\title{
Binge Eating and Binge Eating Disorder in a Small-Scale, Indigenous Society: The View From Fiji
}

\author{
Anne E. Becker, ${ }^{1,2}{ }^{2}$ Rebecca A. Burwell, ${ }^{3}$ Kesaia Navara, ${ }^{4}$ and Stephen E. Gilman ${ }^{5,6}$ \\ ${ }^{1}$ Department of Social Medicine, Harvard Medical School, Boston, Massachusetts \\ ${ }^{2}$ Department of Psychiatry, Massachusetts General Hospital, Boston, Massachusetts \\ ${ }^{3}$ Department of Psychology, University of Denver, Denver, Colorado \\ ${ }^{4}$ Village Health Station, Rararua Village, Fiji \\ ${ }^{5}$ Department of Maternal and Child Health, Harvard School of Public Health, \\ Boston, Massachusetts \\ ${ }^{6}$ Centers for Behavioral and Preventive Medicine, Brown Medical School \\ and The Miriam Hospital, Providence, Rhode Island
}

Accepted 22 April 2003

\begin{abstract}
Objective: Although the cross-cultural prevalence of anorexia and bulimia nervosa has been investigated in multiple studies, little is known about the prevalence and correlates of binge eating and binge eating disorder (BED) cross-culturally. No published studies to date have explored BED in small-scale, indigenous, or developing societies. The current study investigated the prevalence and correlates of binge eating in a community sample of Fijian women living in rural Fiji. Methods: Fifty ethnic Fijian women completed a self-report measure developed for this study on dieting and attitudes toward body shape and change, a Nadroga-language questionnaire on body image, and the Questionnaire on Eating and Weight Patterns-Revised (QEWP-R). Their height and weight were also measured. Patterns of dieting, high body mass index (BMI), and attitudes toward eating and body image were compared between women with and without a history of binge eating. Results: Ten percent of respondents reported at least weekly episodes of binge eating during the past 6 months and 4\% endorsed symptoms consistent with BED. Binge eating in this sample was associated significantly with a BMI value above 35, a history of dieting, and a high concern with body shape. Binge eating was not associated with several markers of acculturation in this sample, although it was associated with a key, nontraditionally Fijian (i.e., acculturated) attitude toward the body. Discussion: Binge eating occurred in a social context with traditions concerning weight and diet widely disparate from Western populations. However, correlates of binge eating in this sample suggest that nontraditional Fijian attitudes toward weight and body shape play a contributory role. (c) 2003 by Wiley Periodicals, Inc. Int J Eat Disord 34: 423-431, 2003.
\end{abstract}

Key words: binge eating; binge eating disorder; cross-cultural prevalence

\footnotetext{
${ }^{*}$ Correspondence to: Anne E. Becker, M.D., Ph.D., Adult Eating and Weight Disorders Program, Massachusetts General Hospital-WAC 816, 15 Parkman St., Boston, MA 02114. E-mail: abecker@partners.org

Published online in Wiley InterScience (www.interscience.wiley.com). DOI: 10.1002/eat.10225

(C) 2003 by Wiley Periodicals, Inc.
} 


\section{INTRODUCTION}

Epidemiologic data on the cross-cultural occurrence and prevalence of anorexia nervosa and bulimia nervosa suggest sociocultural contributions to their etiology. However, little is known about how the sociocultural context may contribute to the development of binge eating and the risk of binge eating disorder (BED). This study examined binge eating and BED in a small-scale, indigenous, society. In Fiji, traditional attitudes toward obesity, overeating, and reshaping of the body have been quite distinctive from the prevailing cultural values placed on thinness and healthful eating in the Western societies in which BED has been described and investigated. The presence of binge eating in a distinctive social context and its emergence in the setting of rapid social transitioning potentially illuminate mechanisms by which cultural factors contribute to the etiology of disordered eating of all types. Most published research on social risk factors for eating disorders has focused on societal pressures to be thin (Garner, Garfinkel, Schwartz, \& Thompson, 1980), media exposure (Groesz, Levine, \& Murnen, 2002), and social transition (Lee, 1998). Other ways in which the social landscape may enhance the risk for excessive concern with body shape and, specifically, for binge eating, have not been well elucidated.

\section{Prevalence of Binge Eating and BED}

The prevalence of binge eating and BED has been assessed in both community samples and in weight control program clinical samples in a variety of Western societies. In the United States, the point prevalence of BED is estimated to be $1.5-4.5 \%$ and $28.8 \%-30.1 \%$ among community and clinical samples, respectively (Smith, Marcus, Lewis, Fitzgibbon, \& Schreiner, 1998; Spitzer et al., 1992, 1993). The point prevalence for binge eating not meeting full criteria for BED (i.e., the number of binge eating episdoes does not meet the frequency criteria) has been estimated to be 2.1-6.3\% among community samples and $37.9 \%$ among clinic, weight control program participants (Spitzer et al., 1992).

Comparative prevalence studies of binge eating and BED among ethnic groups in the United States have suggested an elevation in risk among ethnic minority women. Rates of binge eating and BED are as high or higher among African American women than among Caucasian women in both community and clinic-based samples (Spitzer et al., 1993; Striegel-Moore, Wilfley, Pike, Dohm, \& Fairburn, 2000; Yanovski, Gormally, Leser, Gwirtsman, \& Yanovski, 1994; Yanovski, Nelson, Dubbert, \& Spitzer, 1993). In addition, rates of BED among Latina women are as high or higher than among African American and Caucasian women (Bruce \& Agras, 1992; Fitzgibbon et al., 1998; Smith \& Krejci, 1991). Asian American women have comparable rates of BED to African American and Caucasian women (le Grange, Stone, \& Brownell, 1998). No difference in the rate of binge eating between Native American adolescents and Caucasian adolescents has been found (Smith \& Krejci, 1991).

Internationally, data on binge eating and BED are almost exclusively available for Western populations. The data suggest similar rates of binge eating and BED to community samples in the United States. For example, the rates of BED in community samples are 3.3\% in Austria (in females) (Kinzl, Traweger, Trefalt, Mangweth, \& Biebl, 1999), 1.0\% in Australia (Hay, 1998), 1.5\% in Norway (in females) (Gotestam \& Agras, 1995), 1.5\% in England (in an obese community sample of women; Robertson \& Palmer, 1997), and 0.7\% in France (in females) (Basdevant et al., 1995). The prevalence of binge eating in com- 
munity samples in Europe is slightly higher than that reported in the United Sates. For example, the prevalence ranges from $4 \%$ in Zurich (Vollrath, Koch, \& Angst, 1992) to 12\% in an obese community sample of women in England (Robertson \& Palmer, 1997). However, comparison of rates of binge eating is made difficult by the lack of a standardized definition. We are not aware of any published reports documenting the presence of BED in non-Western, postindustrialized societies. One study that reported the results of a two-stage screening among Chinese undergraduates in Hong Kong detected only two cases ( $N=646$ females) of binge eating in the absence of compensatory behaviors (Lee, 1993). Likewise, we are not aware of any published research investigating the occurrence of BED in small-scale, indigenous societies. Therefore, little known about the prevalence and risk factors for binge eating and BED in non-Western cultures.

\section{Correlates of Binge Eating and BED}

BED and binge eating are associated with a high body mass index (BMI) and obesity (Fairburn et al., 1998; Hay, 1998; Smith et al., 1998; Striegel-Moore, Dohm, et al., 2000; Striegel-Moore, Wilfley, et al., 2000). Excessive concern with weight and shape is also common among individuals with both binge eating and BED (Cargill, Clark, Pera, Niaura, \& Abrams, 1999; Eldredge \& Agras, 1996). In clinic and community samples, binge eating and BED are associated with psychiatric distress, including depression and anxiety symptoms (Fitzgibbon et al., 1998; Smith et al., 1998; Spitzer et al., 1993; StriegelMoore, Dohm, et al., 2000; Streigel-Moore, Wilfley, et al., 2000; Telch \& Stice, 1998; Vollrath et al., 1992), and with increased rates of Axis II disorders (Telch \& Stice, 1998; Yanovski et al., 1993).

BED is also associated with a history of weight fluctuations (Basdevant et al., 1995; Spitzer et al., 1992, 1993; Yanovski, 1993), childhood obesity, and a history of critical comments or teasing by family that contribute to dieting risk (Fairburn et al., 1998), interpersonal problems (Eldredge, Locke, \& Horowitz, 1998), emotional eating (Eldredge \& Agras, 1996), and low self-efficacy (Miller et al., 1999). Similarly, binge eating has been linked with feelings of inferiority (Vollrath et al., 1992).

\section{Study Site}

Fiji is an archipelago of more than 300 islands on the geographic and cultural border of Melanesia and Polynesia. Ethnic Fijians comprise approximately one half of the population of the Republic of Fiji. They have retained many of their traditional lifeways and subsistence patterns despite Fiji's gradual entry into the global economy over the past several decades. Before the 1990s, anorexia and bulimia nervosa were believed to be rare or nonexistent among ethnic Fijians (Becker, 1995). However, an increase in indicators of disordered eating behaviors and attitudes among ethnic Fijian teenage girls has been documented in association with the introduction of television in 1995 and in the setting of other recent rapid social change (Becker, Burwell, Gilman, Herzog, \& Hamburg, 2002). There is no indigenous illness category corresponding to any eating disorder described in the 4th ed. of the Diagnostic and Statistical Manual of Mental Disorders (DSM-IV; American Psychiatric Association [APA], 1994), including BED. However, two locally defined syndromes among the indigenous population, macake (a syndrome chiefly characterized by appetite loss and rhinorrea) and "going thin" (both without a Western nosologic correlate), reflect an enormous social concern with appetite and a fear of weight loss. Cultural norms and social mechanisms that strongly support robust appetites and body shapes have been previously documented. For instance, mealtime structure and 
rhetoric, the language of compliments and insults, and the importance of food presentation and feasts as facilitators of social exchange and networks all support hearty consumption of relatively calorie-dense foods (Becker, 1995). Arguably, these dietary patterns are comparable to what would be considered overeating by most Americans.

The prevalence of overweight and obesity is high in Fiji (Becker, 1995), as it is in many indigenous Pacific Island populations (Hodge, Dowse, Zimmet, \& Collins, 1995). Despite a very high rate of obesity, ethnographic data have demonstrated that body satisfaction is high among ethnic Fijian women (Becker, 1995). However, throughout the Pacific, recent data indicate that body esthetic ideals that once favored robust bodies have been transformed in the setting of other social change (Becker et al., 2002; Ben-Tovim, 1996). Dieting to reduce weight is not an indigenous practice in Fiji, but it has become prevalent among teenage girls following the introduction of televised Western-produced programming. Exercise equipment is promoted heavily on televised advertisements, thereby increasing the level of interest in exercise for health and cosmetic reasons.

\section{METHODS}

\section{Participants}

All available ethnic Fijian adult women within a traditional Fijian village in rural Fiji were recruited for study participation with permission and assistance from the village chief. Women were enrolled consecutively until a sample size of 50 was reached. No census data are available for the number of adult women in this village, but the population of the entire village was 289 in 1996 (Fiji Bureau of Statistics), 2 years before study data were collected. Inclusion criteria specified ethnic Fijian ethnicity, an age range of 18-69 years, and residency in the study village. Subjects who could not understand and speak either the Nadroga dialect or English were excluded from participation.

\section{Procedure and Assessments}

Written informed consent was obtained from all participants. They completed the Questionnaire on Eating and Weight Patterns-Revised (QEWP-R; see Nangle, Johnson, Carr-Nangle, \& Engler, 1994; Spitzer et al., 1992, 1993), as well as a self-report measure developed for this study. The self-report assessed attitudes toward diet, weight, Fijian cultural traditions concerning appetite and food, and media exposure. Both measures were translated into the Nadroga dialect of Fiji, backtranslated into English, and then reviewed by a native speaker for appropriate syntax and grammar. There are no acculturation measures by which to assess departure from tradition in Fijian society. Therefore, the self-report measure was designed to assess assimilation to Western culture as well as acculturation from Fijian tradition in relation to food and bodyrelated traditions. The QEWP-R evaluated binge eating behavior and associated symptomatology. Additional items from a previously developed Nadroga-language questionnaire on body image for this population were used to assess attitudes toward body shape in a more familiar local idiom (see Becker, 1995). A native Nadroga dialectspeaking research assistant was present throughout data collection to respond to subject questions about linguistic and conceptual content. The height and weight of the women were also measured. The study was approved by both the Fiji Research Committee and the Harvard Medical School Committee on Human Studies. 


\section{Data Analyses}

We compared participants with a history of binge eating with those without self-reported binge eating on a range of variables including BMI and attitudes toward eating and body image. To compare the two groups, we used exact chi-square tests for differences in proportions and $t$ tests (with adjustment for unequal variances when appropriate) for differences in means (all $p$ values were two sided). However, the relatively small sample of participants in this study limits the statistical power to detect significant differences. Therefore, we consider the results of significance tests to be provisional and instead place greater emphasis on the nature and direction of differences between respondents with and without a history of binge eating.

\section{RESULTS}

\section{Characteristics of the Sample}

The mean $(S D)$ age of the subjects was 37.5 years old (12.0). All respondents had primary school education to at least the fifth grade and the majority $(n=31[62 \%])$ had some secondary school education. Of these 31 women, 3 had received some postsecondary school education. Eighty-four percent of the respondents resided in a household with a television. Fifty percent of subjects watched television at least three nights weekly. The mean (SD) BMI of the women was $29.4 \mathrm{~kg} / \mathrm{m}^{2}$ (4.8). Forty-four percent $(n=22)$ of the respondents had a BMI of $30 \mathrm{~kg} / \mathrm{m}^{2}$ or greater, thus meeting the criteria for obesity.

Of the 50 respondents, $5(10 \%)$ endorsed symptoms consistent with binge eating (i.e., episodes of eating an unusually large amount of food within a discrete time period and an associated lack of control; APA, 1994) at least weekly during the past 6 months. These 5 women indicated that their binge eating episodes caused them distress. Of these 5,2 (4\% of the entire sample) endorsed a frequency of binge eating and associated symptoms consistent with BED. None of these 5 subjects endorsed purging symptoms or excessive exercise. Although 1 of these subjects endorsed a frequency of 24-hr fasting suggestive of a diagnosis of nonpurging bulimia, she did not meet criteria for nonpurging bulimia based on contradictory responses that indicated she was binge eating on days she said she was also fasting around the clock. This subject was not available for an interview to clarify her inconsistent responses. None of the subjects in the sample endorsed symptoms consistent with a diagnosis of either purging bulimia or anorexia nervosa.

\section{Correlates of Binge Eating}

We investigated whether binge eating among Fijian women would correlate positively with overweight, dieting, body dissatisfaction, and excessive concern with body shape as have been described in Western populations. Mean BMI was similar among non-binge eaters (29.2 \pm 4.7$)$ and binge eaters (31.4 \pm 5.7$)$. However, a self-report of highest lifetime BMI greater than $35 \mathrm{~kg} / \mathrm{m}^{2}$ was significantly more likely among binge eaters $(40 \%)$ than among non-binge eaters $(2.2 \%$; exact $p=.024)$. It is also noteworthy that non-bingeeating respondents underestimated their highest lifetime weight (a mean BMI of $24.8 \pm$ 4.9 vs. the actual BMI at the time of the study, i.e., $29.2 \pm 4.7)$. On the basis of paired $t$ tests 
Table 1. Differences between respondents with and without a history of binge eating on response to selected items in a Nadroga-language questionnaire on body image and attitudes

\begin{tabular}{llcccc}
\hline & $\begin{array}{c}\text { History of Binge } \\
\text { Eating }(n=5) \\
M(S D)\end{array}$ & $\begin{array}{c}\text { No History of Binge } \\
\text { Eating }(n=45) \\
M(S D)\end{array}$ & $t$ & df & $p$ \\
\hline $\begin{array}{l}\text { 1. Satisfication with body } \\
\quad(1=\text { very positive, } 7=\text { very negative) }\end{array}$ & $4.6(2.6)$ & $3.0(1.9)$ & -1.7 & 48 & .098 \\
$\begin{array}{l}\text { 2. Possible to change body shape } \\
\quad(1=\text { very, } 7=\text { not at all) }\end{array}$ & $2.0(1.0)$ & $4.3(2.1)$ & 2.4 & 46 & .022 \\
$\begin{array}{l}\text { 3. Made attempt to change body shape } \\
\quad(1=\text { very much, 7 = not at all) }\end{array}$ & $1.6(0.9)$ & $5.0(2.2)$ & 3.5 & 45 & .001 \\
$\begin{array}{l}\text { 4. Self-critical about body shape } \\
\quad(1=\text { very, } 7=\text { not at all) }\end{array}$ & $1.2(0.4)$ & $3.4(2.1)$ & 6.1 & 29.8 & $<.001$ \\
\begin{tabular}{l} 
5. $\begin{array}{l}\text { Critical about others' body shape } \\
(1=\text { very, } 7=\text { not at all) }\end{array}$ \\
\hline
\end{tabular} & $1.0(0.0)$ & $4.2(2.5)$ & 8.6 & 44 & $<.001$ \\
\hline
\end{tabular}

conducted separately for binge eaters and non-binge eaters, the difference between actual and reported highest-ever BMI was significantly different among non-binge eaters (mean [SD] difference $=-4.6[4.5], t=-6.7, d f=43, p<.001$ ) but not among binge eaters (mean $[S D]$ difference $=8.4[16.9], t=1.1, d f=4, p=.33$ ).

As predicted, a history of dieting was significantly more likely among women who had recurrent episodes of binge eating (80\%) than among women who did not binge eat $(24 \%$; exact $p=.024)$. As seen in other populations, responses to the Nadroga-language questionnaire on body image (Item 4, Table 1) demonstrated that respondents with binge eating manifested significantly increased concern and tendency to be critical regarding their own body shape compared with respondents without binge eating. Study respondents with binge eating were also significantly more likely than those without binge eating to be critical of the body shapes of others (Item 5, Table 1). Consistent with selfreports on history of dieting, respondents with binge eating were significantly more likely to have made an attempt to alter their body shape (Item 3, Table 1). Finally, there was a nonsignificant difference in the expected direction with respondents with binge eating reported more dissatisfaction with their bodies than respondents without binge eating (Item 1, Table 1).

We tested hypotheses that subjects who reported binge eating would depart further from traditional Fijian attitudes and practices than subjects who did not report binge eating. We investigated the association between binge eating and markers of acculturation to Western practices, including educational attainment, television ownership, television viewing frequency, diminished concerns about the general seriousness of or becoming afflicted with the culture-bound syndromes (i.e., macake and going thin), diminished use of traditional remedies to protect a healthy appetite, and reluctance to attend traditional feasts. The last three markers were chosen based on previously demonstrated cultural salience (Becker, 1995). No significant differences were detected between respondents with and without binge eating on these variables. However, there was a significant difference on one important marker of adherence to Fijian tradition, namely, the traditional Fijian attitude that body shape is relatively fixed and thus not responsive to efforts to change it. Respondents with binge eating were significantly more likely than non-binge-eating respondents to believe that it is possible to reshape the body (Item 2, Table 1). 


\section{DISCUSSION}

Prevalence studies of binge-eating disorder have shown occurrence among North American, European, British, and Australian populations, but have not yet investigated its occurence in small-scale, indigenous societies. Demonstrating the occurrence and correlates of binge-eating in such a society-in this case, in an ethnic Fijian population, with widely disparate indigenous practices concerning food and attitudes toward the body and weight-is of interest as a means of elucidating contributions of the social environment to the pathogenesis of this illness. Because previous studies have demonstrated that BED is associated with overweight, dieting, and excessive concern with body shape, local cultural values and behavioral repertoires concerning appropriate food consumption and body practices would appear to be salient to moderating risk for binge-eating as well as possibly mitigating the distress associated with binge-eating disorder.

Notwithstanding the absence of an indigenous Fijian category corresponding to BED, at least 2 women (4\%) in this sample met full DSM-IV criteria for BED. Three additional respondents endorsed symptoms consistent with clinically significant binge eating (i.e., a total of $10 \%$ of the sample). These prevalences are comparable to those reported in a variety of Western populations. As in studies in Western cultural settings, a history of obesity (in the current study, BMI $>35$ or at least Class II obesity), dieting, and an elevated concern with body shape were correlated with binge eating behavior in this sample of 50 Fijian women. Although some specific markers of acculturation and assimilation were generally not associated with binge eating in this study, respondents with binge eating were significantly more likely to endorse nontraditional Fijian dieting behavior as well as the distinctively nontraditional Fijian (i.e., acculturated) attitude that the body can be reshaped through diet and exercise. In addition, the absence of binge eating in this sample was significantly associated with an underestimation of maximum body weight, suggesting a potential link between the traditional comfort and complacency with body shape in Fiji to protection against binge eating.

There is no formal documentation of binge eating among Fijian women preceding recent social and economic changes in Fiji. As a result, it is impossible to know whether binge eating in this sample reflects a long-standing behavioral pattern among Fijian women or whether it is a recent phenomenon precipitated by social transitions that have promoted increased interest in weight loss among Fijians (Becker et al., 2002). Regardless of the timing of the emergence of binge eating among ethnic Fijian women, its presence among ethnic Fijian women is particularly intriguing for several reasons. First, it is striking that the phenomenology of binge eating and its correlates are similar in two very different cultural environments with respect to dietary custom and convention. Fijian cultural values continue to support concern and vigilance for appetite and weight loss in two specific culture-bound syndromes (i.e., macake and going thin). In addition, Fijian custom continues to emphasize feasting and food exchange as the primary means of social exchange. Surprisingly, individuals with binge eating in this sample did not differ from non-binge-eating respondents in their adherence to either of these cultural institutions. Fijian traditions that have supported robust appetites, a generally favorable response to higher body weight, and highly structured and socialized mealtimes have not buffered the occurrence of binge eating and its associated distress. These findings suggest that clinically significant binge eating can emerge in a variety of social contexts, even those markedly disparate from the Western cultural contexts in which it was first 
described. Conversely, a cultural and physical environment that supports relatively high rates of overweight and obesity, calorie-dense diets, sanctioned overeating at frequent feasts and routine meals, and sensitivity to and commentary on the body shapes of others does not appear to promote an unusually high prevalence of binge eating. In addition, it is possible that these and other, as yet unidentified, cultural variables compete to either increase risk for or confer protection from the development of binge eating in this environment in ways that are dissimilar to Western cultural environments, but still produce a similar prevalence of the behavior.

Key similarities between this sample and North American and European samples in the correlates of binge eating (e.g., dieting history and elevated concern with body shape) are comparatively nontraditional pursuits and concerns among Fijians. It is tempting to speculate that the superimposition of cultural support for dieting and excessive concern with body shape on a social environment that promotes calorie-dense diets, overeating, and/or sensitivity to weight changes in others may play a critical contributory role in the development of binge eating in widely disparate cultural contexts. Further studies in small-scale, indigenous and other non-Western societies will be essential to identify features of the social environment that can be modified to protect individuals from binge eating in a variety of cultural settings.

The authors thank the Tui Sigatoka for her gracious assistance in subject recruitment. They are indebted to Dr. Paul Geraghty of the Fijian Dictionary Project for assistance in backtranslation of the assessments for this study and to Linda Park for her help with data management. Funding for this study was provided by The Irene Pollin Fellowship in Memory of Cherry Adler (to A.E.B.) and the Milton Fund (to A.E.B.), both of Harvard Medical School.

\section{REFERENCES}

American Psychiatric Association. (1994). Diagnostic and statistical manual for mental disorders (4th ed.). Washington, DC: Author.

Basdevant, A., Pouillon, M., Lahlou, N., Le Barzic, M., Brillant, M., \& Guy-Grand, B. (1995). Prevalence of binge eating disorder in different populations of French women. International Journal of Eating Disorders, 18, 309-315.

Becker, A.E. (1995). Body, self, and society: The view from Fiji. Philadelphia: University of Pennsylvania Press.

Becker, A.E., Burwell, R.A., Gilman, S.,E., Herzog, D.B., \& Hamburg, P. (2002). Eating behaviours and attitudes following prolonged exposure to television among ethnic Fijian adolescent girls. Brirish Journal of Psychiatry, 180, 509-514.

Ben-Tovim, D.I. (1996). Is big still beautiful in Polynesia? Lancet, 348, 1047-1048.

Bruce, B., \& Agras, W.S. (1992). Binge eating in females: A population-based investigation. International Journal of Eating Disorders, 12, 365-373.

Cargill, B.R., Clark, M.M, Pera, V., Niaura, R.S., \& Abrams, D.B. (1999). Binge eating, body image, depression, and self-efficacy in an obese clinical population. Obesity Research, 7, 379-386.

Eldredge, K.L., \& Agras, W.S. (1996). Weight and shape overconcern and emotional eating in binge eating disorder. International Journal of Eating Disorders, 19, 73-82.

Eldredge, K.L., Locke, K.D., \& Horowitz, L.M. (1998). Patterns of interpersonal problems associated with binge eating disorder. International Journal of Eating Disorders, 23, 383-389.

Fairburn, C.G., Doll, H.A., Welch, S.L., Hay, P.J., Davies, B.A., \& O'Connor, M.E. (1998). Risk factors for binge eating disorder: A community-based, case control study. Archives of General Psychiatry, 55, 425-432.

Fiji Bureau of Statistics 1996 Census of Fiji.

Fitzgibbon, M.L., Spring, B., Avellone, M.E., Blackman, L.R., Pingitore, R., \& Stolley, M.R. (1998). Correlates of binge eating in Hispanic, Black, and White women. International Journal of Eating Disorders, 24, 43-52.

Garner, D.M., Garfinkel, P.E., Schwartz, D., \& Thompson, M. (1980). Cultural expectations of thinness in women. Psychological Reports, 47, 483-491.

Gotestam, G.K., \& Agras, W.S. (1995). General population-based epidemiological study of eating disorders in Norway. International Journal of Eating Disorders, 18, 119-126. 
Groesz, L.M., Levine, M.P., \& Murnen, S.K. (2002). The effect of experimental presentation of thin media images on body satisfaction: A meta-analytic review. International Journal of Eating Disorders, 31, 1-16.

Hay, P. (1998). The epidemiology of eating disorder behaviors: An Australian community-based survey. International Journal of Eating Disorders, 23, 371-382.

Hodge, A.M., Dowse, G.K., Zimmet, P.Z., \& Collins, V.R. (1995). Prevalence and secular trends in obesity in Pacific and Indian Ocean island populations. Source Obesity Research (3 Suppl. 2), 77s-87s.

Kinzl, J.F., Traweger, C., Trefalt, E., Mangweth, B., \& Biebl, W. (1999). Binge eating disorder in females: A population-based investigation. International Journal of Eating Disorders, 25, 287-292.

Lee, S. (1993). How abnormal is the desire for slimness? A survey of eating attitudes and behaviour among Chinese undergraduates in Hong Kong. Psychological Medicine, 23, 437-451.

Lee, S. (1998). Global modernity and eating disorders in Asia. European Eating Disorders Review, 6, $151-153$.

le Grange, D., Stone, A.A., \& Brownell, K.D. (1998). Eating disturbances in white and minority female dieters. International Journal of Eating Disorders, 24, 395-403.

Miller, P.M., Watkins, J.A., Sargent, R.G., \& Rickert, E.J. (1999). Self-efficacy in overweight individuals with binge eating disorder. Obesity Research, 7, 552-555.

Nangle, D.W., Johnson, W.G., Carr-Nangle, R.E., \& Engler, L.B. (1994). Binge-eating disorder and the proposed DSM-IV criteria: Psychometric analysis of the Questionnaire of Eating and Weight Patterns. International Journal of Eating Disorders, 16, 147-157.

Robertson, D.N., \& Palmer, R.L. (1997). The prevalence and correlates of binge eating in a British community sample of women with a history of obesity. International Journal of Eating Disorders, 22, 323-327.

Smith, D.E., Marcus, M.D., Lewis, C., Fitzgibbon, M., \& Schreiner, P. (1998). Prevalence of binge eating disorder, obesity, and depression in a biracial cohort of young adults. Annals of Behavioral Medicine, 20, $227-232$.

Smith, J.E., \& Krejci, J. (1991). Minorities join the majority: Eating disturbances among Hispanic and Native American youth. International Journal of Eating Disorders, 10, 179-186.

Spitzer, R.L., Devin, M., Walsh, B.T., Hasin, D., Wing, R., Marcus, M., Stunkard, A., Wadden, T., Yanovski, S., Agras, S., Mitchell, J., \& Nonas, C. (1992). Binge eating disorder: A multisite field trial of the diagnostic criteria. International Journal of Eating Disorders, 11, 191-203.

Spitzer, R.L., Yanovski, S., Wadden, T., Wing, R., Marcus, M.D., Stunkard, A., Devlin, M., Mitchell, J., Hasin, D., \& Horne, R.L. (1993). Binge eating disorder: Its further validation in a multisite study. International Journal of Eating Disorders, 13, 137-153.

Striegel-Moore, R.H., Dohm, F.A., Solomon, E.E., Fairburn, C.G., Pike, K.M., \& Wilfley, D.E. (2000). Subthreshold binge eating disorder. International Journal of Eating Disorders, 27, 270-278.

Striegel-Moore, R.H., Wilfley, D.E., Pike, K.M., Dohm, F., \& Fairburn, C.G. (2000). Recurrent binge eating in black American women. Archives of Family Medicine, 9, 83-87.

Telch, C.F., \& Stice, E. (1998). Psychiatric comorbidity in women with binge eating disorder: Prevalence rates from a non-treatment-seeking sample. Journal of Consulting and Clinical Psychology, 66, 768-776.

Vollrath, M., Koch, R., \& Angst, J. (1992). Binge eating and weight concerns among young adults: Results from the Zurich cohort study. British Journal of Psychiatry, 160, 498-503.

Yanovski, S. (1993). Binge eating disorder: Current knowledge and future directions. Obesity Research, 1, 306-318.

Yanovski, S.C., Gormally, J.F., Leser, M.S., Gwirtsman, H.E., \& Yanovski, J.A. (1994). Binge eating disorder affects outcome of comprehensive very low-calorie diet treatment. Obesity Research, 2, 205-212.

Yanovski, S.C., Nelson, J.E., Dubbert, B.K., \& Spitzer, R.L. (1993). Associations of binge eating disorder and psychiatric comorbidity in obese subjects. American Journal of Psychiatry, 150, 1472-1479. 